\title{
Semantic Web Technology: A Strategic Approach to Intelligence
}

\author{
Sudeshna Chakraborty \\ Astt. Professor, SD Inst of Mgmt \& \\ Tech, Faridabad, India \\ H. No. 1424, Sector 28 \\ Faridabad-121008, Haryana, India
}

\author{
Rekha Mittal \\ Astt. Professor, SD Inst of Mgmt \& \\ Tech, Faridabad, India \\ H. No. 2485, Sector $7 A$ \\ Faridabad-121006, Haryana, India
}

\begin{abstract}
Presently besides the classic "Web of documents" it is now required to build a technology stack to support a "Web of data," the sort of data we find in databases. The vital goal of the Web of data is to enable computers to do more useful work and to build up systems that can support trusted interactions over the network. The title "Semantic Web" refers to the Web of allied data. Semantic Web technologies enable people to create data stores on the Web, build vocabularies, and provide protocols for handling data. Linked data are empowered by technologies such as RDF, SPARQL, and OWL. This paper primarily put the idea behind the understanding by the computer. The goal is to develop techniques for automatic creation of intelligent web applications which collect information from many different sources, combine information, and present it to users in a meaningful way. This paper proposes a model which has been implemented in an internet setup. The aim of the model is to assemble an application for users where the user lists a set of topic names (s) he is interested in, and the system aids in constructing the "best" usertailored structure, where "best" is characterized in terms of what the user knows about topics.
\end{abstract}

\section{General Terms}

Protocols, Internet, Web of data

\section{Keywords:-}

RDF, SPARQL, OWL

\section{INTRODUCTION}

Today there are plenty of web tools available on the net. The word semantic stands for the meaning of something.

The Semantic Web $=$ a Web with a meaning.

The Semantic Web is a web that is able to describe things in a way that computers can understand.

- Pink Floyd were an English rock band who earned recognition for their psychedelic rock music in the late 1960s

- Richard William was a member of the Pink Floyd.

- "Meddle" was recorded by the Pink Floyd.

Sentences like the ones above can be understood by people. We have to put the idea behind the understanding by the computer. Statements are built with syntax rules. The syntax of a language defines the rules for building the language statements. But how can syntax become semantic?

This is what the Semantic Web is all about, describing things in a way that computer applications can understand it. The Semantic Web is not about links between web pages.

It describes the relationships between things (like $\mathrm{A}$ is a part of $\mathrm{B}$ and $\mathrm{Y}$ is a member of $\mathrm{Z}$ ) and the properties of things (like size, weight, age, and price.

\section{Personal Information disaster}

There are lots of ways in which our machines can use data when they can understand it. When my calendar understands dates, it can warn me when an appointment is coming up.

When my Global Position System device understands latitude and longitude, it can show me the way to where I should be.

When my address book understands that something is a phone number or an email address, it can set up communication with a person with a click

But the reality is something different!!

\section{Enterprise Application Integration}

- $\quad$ Trying to connect the various data-handling applications on which our company depends

- If we have $\mathrm{N}$ applications which run our company, there are of order $\mathrm{N}^{2}$ ways in which we may want to connect them together .

- If all the applications use XML, for each way in which they need to be linked, someone has to create an XML to XML bridge

- If we take XML files from two different applications, we can't just merge them.

$\mathrm{XML}$ is not enough. The magnificent challenge is to enhance the collective IQ of the organization or the society.

\section{Review Literature}

\subsection{What is semantic web?}

It is an extension of the current web in which information is given well-defined meaning, better enabling computers and people to work in cooperation."

Tim Berners-Lee, James Handler, Ora Lassila 


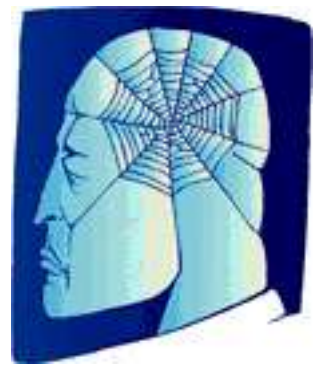

It consists of resources $=$ nodes $=$ subject which have properties $=$ predicate

Which have values $(=$ nodes, strings $)=$ object

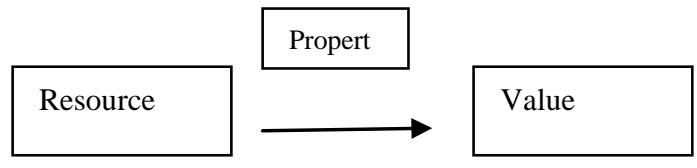

Example of RDF Model applications, enterprises, communities, and people, independent of platform and software. The web of data that can be processed by machines, enabling them to interpret, combine and use Web data. The Semantic Web offers a uniform way of identifying and describing resources in a distributed environment, and thus increases the interoperability between applications.

RDF and OWL are key Semantic Web technologies.

The term has occasionally been used ambiguously to refer as to either an individual work that can be manipulated automatically, or by following hyperlinks to definitions of key terms and rules for reasoning about them logically. The resulting infrastructure will spur the development of automated Web.

\section{RDF}

\subsection{RDF Model}

RDF Model can be thought of as a simplified XML Info set

All one needs to do is move up a thin layer of interoperability in web. Unstructured data can also adopt a relational model.

The relational language for data on the Net is called RDF

- RDF information can have more or less same structure like XML

- RDF data is made up of statements where each statement expresses the value of one property of something

- All the relational database ideas work - for example, joins and views.

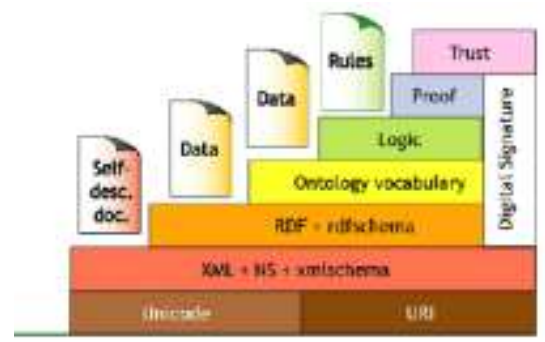

The model is domain-neutral, application-neutral and ready for internationalization (i18n)

The model can be viewed as directed, labeled graphs or as an object-oriented model (object/attribute/value)

RDF data model is an abstract, conceptual layer independent of XML.

\section{Example of RDF Model}

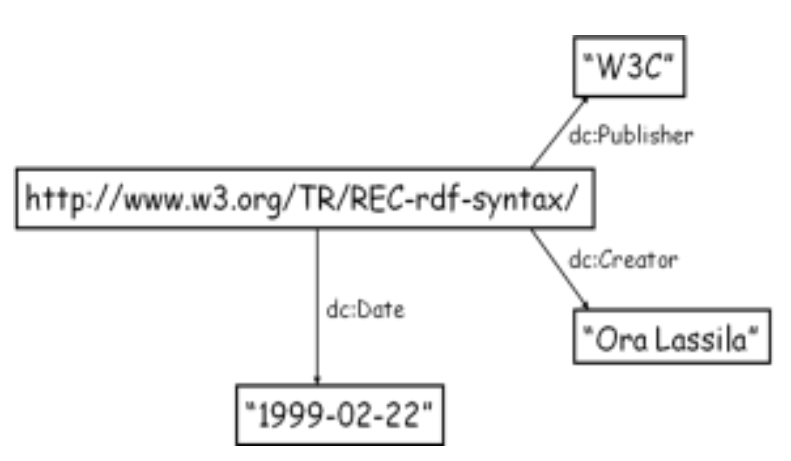

\subsection{Higher-order statements}

- One can make RDF statements about other RDF statements

Example: "Ralph believes that the web contains one billion documents"

- Higher-order statements

Allow us to express beliefs (and other modalities) are important for trust models, digital signature etc.

\subsection{Description}

<rdf:Description about="http://www.openhealth.org/RDF/rdfExtractify.xsl">

$<$ dc: author $>$ Jonathan Borden</dc:author $>$

$<\mathrm{dc}$ : description>An XSLT based parser which converts an arbitrary XML document into RDF Statements $</$ dc: description $>$

$</$ rdf: Description>

about="http://www.openhealth.org/RDF/rdfExtractify.xsl">

$<\mathrm{dc}$ : author $>$ Jonathan Borden</dc:author $>$

$<\mathrm{dc}$ : description>An XSLT based parser which converts an arbitrary XML document into RDF Statements $</$ dc:description $>$

$</$ rdf:Description>

\subsection{Statement}

<rdf:Statement>

$<$ rdf:subject 
resource="http://www.openhealth.org/RDF/rdfExtractify.xsl">

$<$ rdf: predicate resource="http://www.purl.org/dublincore\#author">

$<$ rdf:object $>$ Jonathan Borden $</$ rdf:object $>$

$</$ rdf:Statement $>$

\subsection{Resource Description Framework Schema}

- Allows definition of new classes of resources and properties

- Can be considered a simple Knowledge Representation/Ontology Language.

\subsection{Classes}

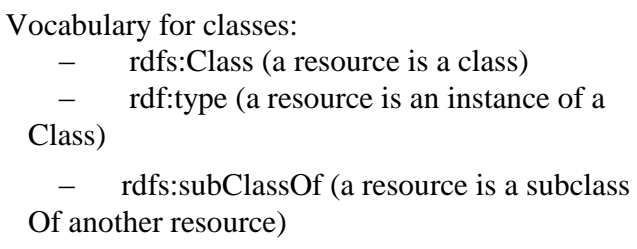

\subsection{Hierarchy of classes}

Hierarchy of properties (like, father of is specialization of parent of)

Domain and Range restrictions of the properties

No limitations in the levels of instantiation -e.g. one resource could be a class itself.

\subsection{Vocabulary for properties:}

- $\quad$ rdf:Property (a resource is a property)

- rdfs:domain(denotes the first component of a property)

- rdfs:range (denotes the second component

Of a property)

- rdfs:subPropertyOf (expresses ISA

Between properties)

Example of Hierarchy of class

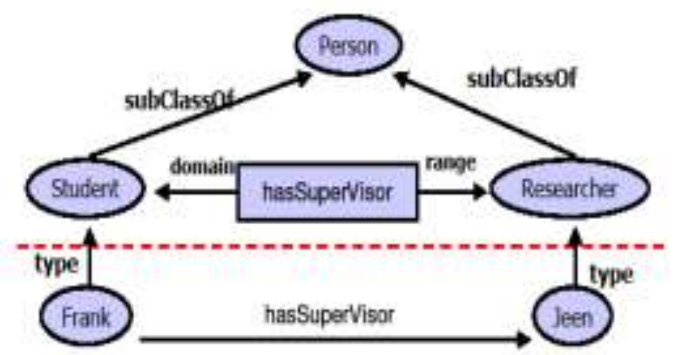

\section{OWL:-The Web Ontology Language}

Ontology is a specification of a conceptualization. It was designed to provide a general way to process the content of web information (instead of displaying it).OWL was designed to be read by computer applications (instead of humans).

\subsection{OWL is Different from RDF}

OWL and RDF are much of the same thing, but OWL is a stronger language with greater machine interpretability than RDF.OWL comes with a larger vocabulary and stronger syntax than RDF.

\subsection{The Semantic Web and Ontology}

Backbone of Semantic Web is Ontology

Formal structures supporting knowledge sharing and reuse

Can represent explicitly the semantics of structured and semi structured information

\subsection{Origin of Ontology}

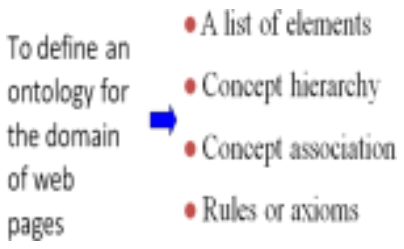

This can enable sophisticated automatic support for acquiring, maintaining and accessing information.

\subsection{What is the Advantage of using Ontology}

- It improves the quality of the output in many ways.

- It becomes possible to capture the contextual relationship among various components within the document

- It leads to better understanding of the information contained within the document.

- This additional information can be used in other processes, such as document categorization and contextual search.

\subsection{Future Work}

- It is assumed that the future of mobile browsing lies in the adoption of semantic web technology.

- Before that realizes, the proposed approach offers a workable compromise to generate high fidelity reauthored web pages.

- This is an exploratory paper offering a specific pathway to the future of web page re-authoring provided accurate layout information is available. 
- Currently, it is beyond the capability of any algorithm to achieve this level of accuracy. However, approximations to that accuracy are attainable and even practical. It will be interesting to discuss other possibilities in this space.

\subsection{Why have Ontology become Popular?}

- Computers are now entry points into a worldwide network of information exchange and business transactions

- Support is required for the exchange of data, information and knowledge

- Ontology's offer a:

Shared understanding of some domain

I. That can be communicated between people and application system.

II .Way to describe the structure and semantics of exchange

\subsection{Markup Ontology Languages}

These languages use a markup scheme to encode knowledge, most commonly XML DAML+OIL is a successor language to DAML and OIL that combines features of both.

\section{Ontology Inference layer (OIL)}

OIL is based on concepts developed in Description Logic (DL) and frame based systems and is compatible with RDFS.

\section{Extending Ruby on Rails for Semantic Web(A case Study)-}

Ruby on Rails is an agile development platform for Web applications. It provides solutions for rapid prototyping and is supported by an active community. Ruby on Rails is currently a popular platform for development of Web 2.0 applications.

The Semantic Web is a web of data that can be processed by machines, enabling them to interpret, combine and use Web data. The Semantic Web offers a uniform way of identifying and describing resources in a distributed environment. SWORD is a ruby-on-rails plug-in for Semantic Web development. SWORD includes scaffold generators for models, controllers, and views. The generators take into account that on the semantic web, data, schemas, and views are distributed and decentralized: running applications means fetching and integrating data and views from various online sources and thus increases the interoperability between applications.

\section{Bibliography Data Ontology}

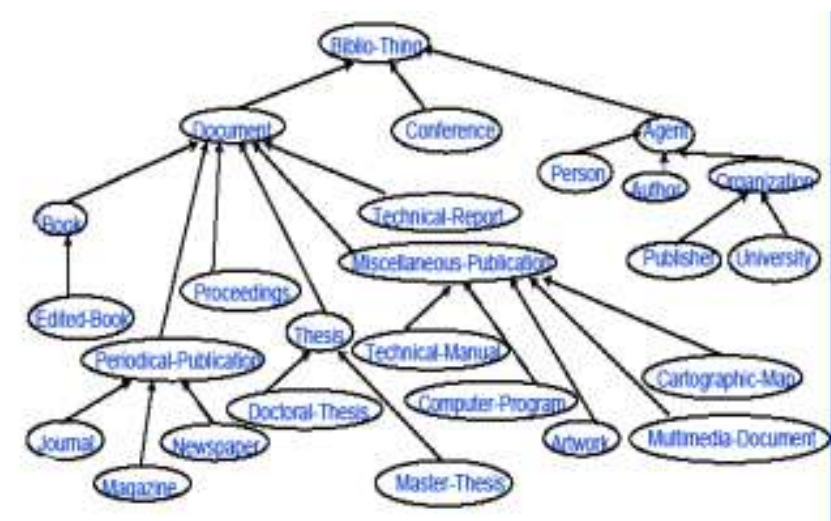

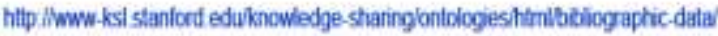

\section{Semantic Web Features}

- Semantic Web Services are far from the end of the Semantic Web vision.

- There's quite a bit of interesting research and development still going on.

- Four major issues:

$$
\begin{array}{ll}
\text { 1. Aggregation } \\
\text { 2. Security } \\
\text { 3. Logic } \\
\text { 4. Agents }
\end{array}
$$

\subsection{Aggregation}

- Systems like Google aggregate the many pages of the Web into a cohesive database you can search.

- Developers are working on similar systems for RDF data, to create a sort of Semantic Google.

- There are two main approaches:

(a) The centralized one, where all the data is collected onto one system;

(b) Decentralized one, where the data is organized across many computers, and each taking responsibility for a different portion.

\subsection{Security}

- $\quad$ RDF builds a "Web of Trust", taking into account who you trust and how much you trust them.

- By connecting these statements with everyone else's, it builds a Web that interconnects almost everyone

- Then it tries to pick statements from people you trust -those closest to you in the Web of Trust.

- RDF also uses digital signatures to ensure that the triples were actually said by who they're claimed to be 
said by.

\subsection{Logic}

- Logic provides a way to express rules that take in triples and output new ones in RDF.

- It's based on the old field of symbolic logic, and has strong mathematic backings.

- Inference Engines are the tools that take in rules and data and process them, writing out the resulting RDF triples.

- Programming using rules is what's called declarative programming.

\subsection{Agents (1)}

- Agents are pieces of software that work autonomously and proactively.

- A personal agent on the Semantic Web will

(a) receive preferences from the person

(b) Seek information from web sources

(c) Communicate with other agents,

(d) Compare information about user requirements and preferences

(e) Select certain choices

(f) Give answers to the users

\section{Agents (2)}

- Semantic Web agents will make use of following technologies

(a) Metadata to identify extract information from web sources

(b) Ontology to assist in web searches, to interpret retrieved information, and to communicate with other agents

(c) Logic used for processing retrieved information and for drawing conclusions E.g. Travel Agent

\section{Conclusion: The Grand Vision}

1. The ease with which your PDA, your laptop, your desktop, your server, and your car will communicate with each other.

2. The automation of corporate decisions that previously had to be laboriously hand-processed. The ability to assess the trustworthiness of documents on the Web.

3. The remarkable ease with which we'll be able to find the answers to our questions.

4. The ability to assess the trustworthiness of documents on the Web.

5. The remarkable ease with which we'll be able to find the answers to our questions.

6. An effective Search engine module has been built that returns content pertaining to a given topic search string. The search result is ordered in terms of complexity and also obeys a ranking based on closeness of search result to search string.

7. "The possibilities are endless, and even if we don't ever achieve all of them, the journeys will most Meta data about metadata are represented by modeling RDF in RDF itself.

\section{ACKNOWLEDGMENTS}

Our special thanks to Dr. Shri Subhash Banerjee, Shri Sandeep Sharma \& Ms Lathka Malik who have contributed towards providing a platform for development of the article.

\section{FURTHER WORK}

The Semantic Web is scattered \& decentralized-- no one organization or individual has control over all of its rules, protocols and content.

This system can serve as the first step toward formulating and solving what maybe a very large number of practical research problems about web semantic.

This application maybe enhanced further to support file formats other than HTML.

Another research direction is to introduce multiple experts, and handle opinion conflict between experts.

In the future, off-the-shelf software might include options for accumulating metadata when creating new documents, but that tool still might not make the project feasible on a larger scale.

\section{REFERENCES}

[1] A Semantic Web Primer, Grigoris Antoniou and Frank van Harmelen, ISBN 0-262-01210-3, 2004, the MIT Press

[2] An introduction to http://www.ibm.com/developerworks/library/w-rdf/

[3] Practical RDF Tools Towards a Semantic Web, http://www.dajobe.org/talks/kt2001/index.html

[4] Portege Home Page, http://protege.stanford.edu/publications/ontology_development/on tology101.html

[5] Semantic Web, W3C http://www.w3c.org/2001/sw/

[6] The Semantic Web, Scientific American, May 2001

[7] Web Engineering: A Practitioner's Approach by Roger Pressman, David Lowe. 